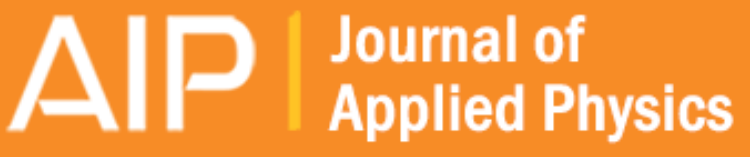

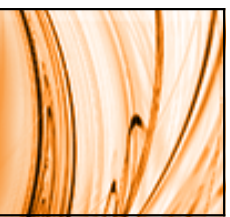

\section{Multiferroic composites for magnetic data storage beyond the super-paramagnetic limit}

M. M. Vopson, E. Zemaityte, M. Spreitzer, and E. Namvar

Citation: Journal of Applied Physics 116, 113910 (2014); doi: 10.1063/1.4896129

View online: http://dx.doi.org/10.1063/1.4896129

View Table of Contents: http://scitation.aip.org/content/aip/journal/jap/116/11?ver=pdfcov

Published by the AIP Publishing

\section{Articles you may be interested in}

Thermally induced error: Density limit for magnetic data storage

Appl. Phys. Lett. 100, 102402 (2012); 10.1063/1.3691196

FePt - TiO 2 exchange coupled composite media with well-isolated columnar microstructure for high density magnetic recording

J. Appl. Phys. 107, 123915 (2010); 10.1063/1.3437044

Microwave assisted magnetization reversal in composite media

Appl. Phys. Lett. 94, 202509 (2009); 10.1063/1.3133354

Effect of orientation on the thermal stability in advanced metal particulate tapes

J. Appl. Phys. 103, 093904 (2008); 10.1063/1.2913319

Composite free layer for high density magnetic random access memory with lower spin transfer current Appl. Phys. Lett. 89, 152509 (2006); 10.1063/1.2361280

\section{HIDEN}

\section{Instruments for Advanced Science}

Contact Hiden Analytical for further details: w www.HidenAnalytical.com E info@hiden.co.uk CLICK TO VIEW our product catalogue

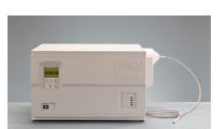

Gas Analysis

dynamic measurement of reaction gas streams catalysis and thermal analysis molecular beam studies

dissolved species probes

fermentation, environmental and ecological studies

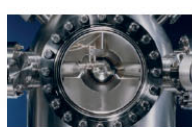

Surface Science

UHVTPD

SIMS end point detection in ion beam etch

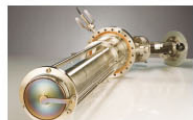

Plasma Diagnostics - plasma source characterization etch and deposition process reaction kinetic studies analysis of neutral and radical species

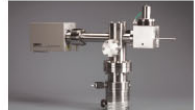

Vacuum Analysis partial pressure measurement and control of process gases reactive sputter process contro , vacuum diagnostics Tacum coating process monitorin 


\title{
Multiferroic composites for magnetic data storage beyond the super-paramagnetic limit
}

\author{
M. M. Vopson, ${ }^{1, a)}$ E. Zemaityte, ${ }^{1}$ M. Spreitzer, ${ }^{2}$ and E. Namvar ${ }^{1}$ \\ ${ }^{1}$ Faculty of Science, University of Portsmouth, SEES, Portsmouth PO1 3QL, United Kingdom \\ ${ }^{2}$ Institut Jožef Stefan, Jamova 39, 1000 Ljubljana, Slovenia
}

(Received 1 July 2014; accepted 9 September 2014; published online 18 September 2014)

\begin{abstract}
Ultra high-density magnetic data storage requires magnetic grains of $<5 \mathrm{~nm}$ diameters. Thermal stability of such small magnetic grain demands materials with very large magneto-crystalline anisotropy, which makes data write process almost impossible, even when Heat Assisted Magnetic Recording (HAMR) technology is deployed. Here, we propose an alternative method of strengthening the thermal stability of the magnetic grains via elasto-mechanical coupling between the magnetic data storage layer and a piezo-ferroelectric substrate. Using Stoner-Wohlfarth single domain model, we show that the correct tuning of this coupling can increase the effective magnetocrystalline anisotropy of the magnetic grains making them stable beyond the super-paramagnetic limit. However, the effective magnetic anisotropy can also be lowered or even switched off during the write process by simply altering the applied voltage to the substrate. Based on these effects, we propose two magnetic data storage protocols, one of which could potentially replace HAMR technology, with both schemes promising unprecedented increases in the data storage areal density beyond the super-paramagnetic size limit. (C) 2014 AIP Publishing LLC.
\end{abstract}

[http://dx.doi.org/10.1063/1.4896129]

\section{INTRODUCTION}

Despite major advances in alternative data storage technologies such as solid state and optical data storage, magnetic recording remains the dominant information storage technology today. However, further increases in magnetic storage densities require additional shrinking of magnetic grain size. Depending on the magnetic material, below a specific critical volume, a magnetic grain enters the superparamagnetic regime at room temperature (RT). Physically this occurs when the thermal Boltzmann energy at RT $(\mathrm{T} \approx$ $300 \mathrm{~K}$ ) is comparable to the magneto-crystalline energy $\mathrm{K}_{\mathrm{a}} \mathrm{V}$ $\approx \mathrm{k}_{\mathrm{b}} \mathrm{T} \approx 25 \mathrm{meV}$, where $\mathrm{K}_{\mathrm{a}}$ is the magneto-crystalline anisotropy constant, $\mathrm{V}$ is the magnetic nano-grain volume, $\mathrm{k}_{\mathrm{b}}$ is Boltzmann constant, and $\mathrm{T}$ is the temperature. In general, a magnetic recording thin film medium is thermally stable for $10 \mathrm{yr}$, at $\mathrm{RT}$ when the ratio $\mathrm{K}_{\mathrm{a}} \mathrm{V} / \mathrm{k}_{\mathrm{b}} \mathrm{T} \geq 40$. $^{1,2}$

Hence, as $\mathrm{V}$ decreases, the thermal stability condition is met by increasing the $\mathrm{K}_{\mathrm{a}}$ parameter. ${ }^{3}$ This has been successfully achieved by developing large magnetic anisotropy materials and by changing the geometry of the magnetic recording from longitudinal data storage to perpendicular data storage. There are, however, two major obstacles hindering further progress of this technology:

(i)

Smaller grains made of very large magneto-crystalline magnetic materials are hard to magnetize using standard write head technology and they require Heat Assisted Magnetic Recording (HAMR) heads, as first proposed by Fujitsu in $1996^{2,4-6}$

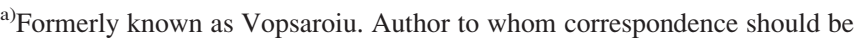
addressed. Electronic mail: melvin.vopson@port.ac.uk.
}

(ii) Smaller grains made of weaker magneto-crystalline materials are not thermally stable at room temperature and the digital data gets thermally self-erased.

In order to overcome these issues, Skumryev et al. proposed to use the exchange bias effect between ferromagnetic and anti-ferromagnetic nano-structures to "beat" the superparamagnetic limit of Co nanoparticles, ${ }^{7}$ an idea also endorsed by Eisenmenger and Schuller. ${ }^{8}$

The magnetic data storage industry has adopted a modified version of this idea, in which the super-paramagnetic limit was overcome by developing anti-ferromagnetic coupled (AFC) recording media instead of exchanged coupled media. ${ }^{9}$ This allowed considerable increases of areal density, but the method is, unfortunately, effective only in the case of longitudinal magnetic recording. With the introduction of perpendicular magnetic recording, the AFC media is no longer practical. More recently, Kim et al. demonstrated experimentally a novel concept of controlling super-paramagnetism via the magnetoelectric effect in multiferroics. ${ }^{10}$ In their study, the multiferroic composite system consisted of $\mathrm{Ni}$ magnetic nanoparticles strain coupled to a piezo-electric $\left[\mathrm{Pb}\left(\mathrm{Mg}_{1 / 3} \mathrm{Nb}_{2 / 3}\right) \mathrm{O}_{3}\right]_{(1-\mathrm{x})^{-}}$ $\left[\mathrm{PbTiO}_{3}\right]_{\mathrm{x}}(\mathrm{PMN}-\mathrm{PT}, \mathrm{x}=0.3)$ substrate. Unpoled substrate left the $\mathrm{Ni}$ nanoparticles in super-paramagnetic state, while the application of $0.4 \mathrm{MV} / \mathrm{m}$ electric field switched the Ni particles to magnetic mono-domain state. These results were interpreted through the occurrence of the strain-mediated magneto-electric coupling between the Ni particles and the piezosubstrate, which, according to the authors, contributes with an additional voltage-induced magneto-elastic energy term to the total energy.

In this paper, we follow up on this study and we propose a multiferroic composite magnetic data storage medium for areal densities beyond the super-paramagnetic limit. The 
potential of this concept has been investigated theoretically using a Stoner-Wohlfarth (S-W) magnetization reversal model, which has been modified to account for the additional electric induced elasto-mechanical coupling in multiferroic materials. The proposed structure (see Fig. 1) consists of a thin film patterned magnetic data storage medium. ${ }^{11}$ The novelty is to deposit the patterned grains onto an active piezo-substrate. The system magnetic grain-piezo-substrate forms a so-called multiferroic composite structure, ${ }^{12-15}$ which displays electrically induced elasto-mechanical coupling. ${ }^{16}$ It is this electrically activated elasto-mechanical coupling that can be utilized to alter the magnetic properties of the magnetic grains, as demonstrated in Sec. II and also recently published in similar studies. ${ }^{17,18}$ Although we refer to a patterned magnetic recording medium, this approach is also valid for polycrystalline magnetic thin films.

\section{THEORY}

We assume that each individual magnetic grain is a uniaxial single domain particle and can be described well in the $\mathrm{S}-\mathrm{W}$ formalism. We also assume that the grains separation distance is large enough to ensure that no exchange or magneto-static inter-grain interactions are present. Hence, analyzing the behavior of an individual constituent particle we can get an insight of the overall mechanism governing the entire sample.

If $\varphi$ is the angle between the applied field and the magnetic easy axis (EA), $\beta$ is the angle between the applied external stress and the EA, and $\theta$ is the angle between the magnetization and the EA (see Fig. 1(b), then for a magnetic particle of volume $\mathrm{V}$, the total energy under applied magnetic field and external stress is

$$
W_{t o t}=K_{a} V \sin ^{2} \theta-H M_{S} V \cos (\varphi-\theta)-\frac{3}{2} \lambda_{s} \sigma_{1} V \sin ^{2}(\beta-\theta),
$$

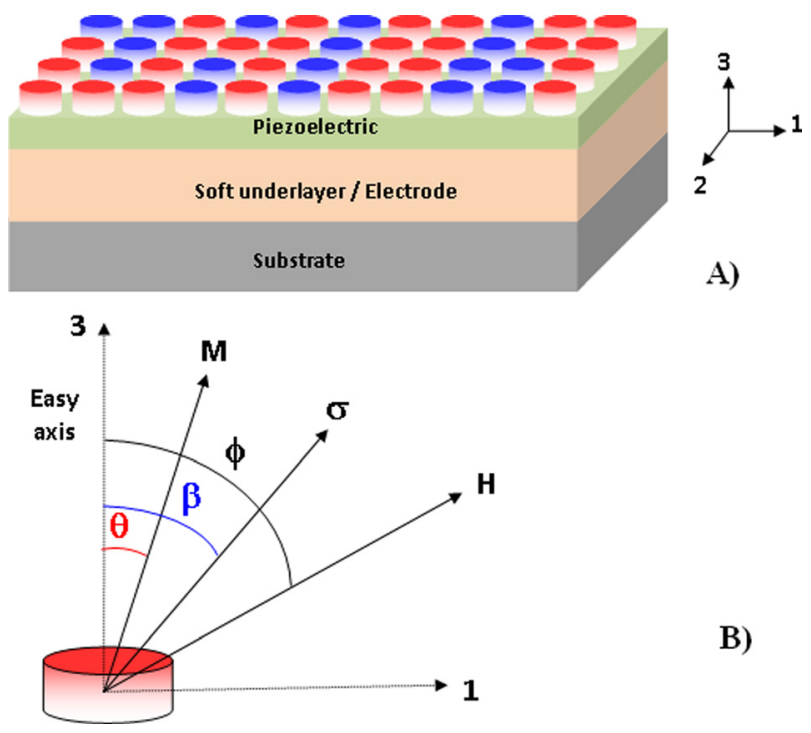

FIG. 1. (a) Schematic of a patterned magnetic recording medium in which magnetic bits form a strain-mediated multiferroic system with the piezoelectric substrate; (b) Uni-axial single domain magnetic particle in applied $\mathrm{H}$ field and $\sigma$ stress induced by the piezo-substrate when electrically activated. where the first term represents the magneto-crystalline anisotropy energy and $\mathrm{K}_{\mathrm{a}}$ is the anisotropy constant, the second term represents the magnetic potential energy due to the applied $\mathrm{H}$ magnetic field and $\mathrm{M}_{\mathrm{s}}$ is the saturation magnetization, and the third term is the magneto-elastic energy for a uni-axial particle where $\lambda_{\mathrm{s}}$ is the saturation magneto-striction coefficient.

The applied stress results from the deformation transferred to the magnetic grains by the piezo-substrate when electrically activated by the application of an electric field/ voltage. Assuming that the ferroelectric substrate is poled in the 3 directions (out of plane), a stress will result in the plane of the magnetic film due to $d_{31}$ piezo-electric coupling, which is given by $\sigma_{1}=\mathrm{Yd}_{31} \mathrm{E}_{3}=\mathrm{Yd}_{31} \mathrm{~V}_{3} / \mathrm{t}_{\mathrm{e}}$, where $\mathrm{t}_{\mathrm{e}}$ is the thickness of the substrate, $d_{31}$ piezo-electric coefficient, $Y$ is the Young's modulus of the ferromagnetic grain, and $V_{3}$ is the amplitude of the applied voltage. We now impose the stress angle along 1 axis $(\beta=\pi / 2)$ and the external magnetic applied field at an angle $\pi$ to the easy axis in order to produce its reversal $(\varphi=\pi)$. With these restrictions imposed, we determine the equilibrium position of the magnetization vector by imposing the condition: $\mathrm{dW}_{\text {tot }} / \mathrm{d} \theta=0$. From this condition, we determine the magnetic anisotropy field as the field required to produce, at equilibrium, a $\pi$ rotation of the $\mathrm{M}_{\mathrm{s}}$ from the EA direction. The following relation for the anisotropy field is obtained:

$$
H_{a}=\frac{1}{M_{s}} \cdot\left(2 K_{a}+\frac{3 \lambda_{s} Y d_{31} V_{3}}{t_{e}}\right) .
$$

It can seen that in the case of a zero applied stress, the anisotropy field is given by the well-known relation: $\mathrm{H}_{\mathrm{a}}=2 \mathrm{~K}_{\mathrm{a}} / \mathrm{M}_{\mathrm{s}}$. However, in the case of an active piezo-substrate inflicting a longitudinal strain to the magnetic grains, we expect a change of the magnetic anisotropy field when $\mathrm{V}_{3} \neq 0$. For a given applied voltage polarity, which is predefined by the poling procedure of the piezo-substrate, relation (2) indicates that the anisotropy field can be both increased and decreased depending on the materials parameters that dictate the signs of $\lambda_{\mathrm{s}}$ and $\mathrm{d}_{31}$, as also shown by Streubel et al. in studies of $\mathrm{Ni} / \mathrm{BaTiO}_{3}$ composite multiferroics. ${ }^{19}$ Assuming a negative applied voltage, a reduction in the anisotropy field can be achieved when both magneto-striction coefficient and the piezoelectric coefficient have the same sign (either negative or positive). When the two parameters have opposing signs, the effect is an increase in the anisotropy field. For a positive applied voltage the effect is reversed. The propagation of material parametric signs into the magnetic anisotropy field of the magnetic nano-particles is well represented in Table I.

TABLE I. Expected trends in the effective magnetic anisotropy field for positive and negative applied voltages as a function of the sign of the magneto-striction and piezoelectric coefficient of the magnetic and electric constituent phases of a laminated multiferroic composite, respectively.

\begin{tabular}{lcccc}
\hline \hline$\lambda_{\mathrm{s}}$ & + & + & - & - \\
$\mathrm{d}_{31}$ & + & - & + & - \\
$\mathrm{H}_{\mathrm{a}}$ at $-\mathrm{V}_{3}$ & Decreases & Increases & Increases & Decreases \\
$\mathrm{H}_{\mathrm{a}}$ at $+\mathrm{V}_{3}$ & Increases & Decreases & Decreases & Increases \\
\hline \hline
\end{tabular}


Obviously, thermal stabilization of a nano-structured magnetic recording medium can be achieved when $\mathrm{H}_{\mathrm{a}}$ increases with the applied voltage, so according to Eq. (2) and Table I, this requires piezoelectric and magneto-strictive coefficients of opposite signs for negative applied voltage and of the same signs for positive applied voltage, respectively.

To better understand the physics behind this effect, let us rewrite (2) in terms of an effective anisotropy field constant

$$
H_{a}=\frac{2 K_{a}^{e f f}}{M_{s}} .
$$

From (2) and (3) we can deduce the effective anisotropy field constant as

$$
K_{a}{ }^{e f f}=K_{a}+\frac{3 \lambda_{s} Y d_{31} V_{3}}{2 t_{e}} .
$$

We now can attribute the anisotropy field variations to the changes in the effective anisotropy field constant of the coupled multiferroic system: magnetic grain-piezoelectric substrate. Relation (4) shows that the effective magnetic anisotropy constant can be either increased or decreased depending on the choice of materials and voltage polarity. This has a direct consequence on the thermal stability of the magnetic medium and it can be utilized to thermally stabilize super-paramagnetic particles for ultra high-density magnetic data storage, as also proposed recently in other similar studies. ${ }^{18,20-22}$

\section{MODELING RESULTS AND DISCUSSIONS}

At zero applied magnetic fields the relaxation time $(\tau)$ is given by the well known Arrhenius-Neel equation

$$
\frac{1}{\tau} \cong \nu_{0} e^{-\frac{K a e f f_{V}}{k_{b} T}},
$$

where $\nu_{0}$ is the magnetization attempt frequency to overcome the energy barrier and it is approximately $\nu_{0} \approx 10^{9} \mathrm{~Hz}$. Hence, the magnetic relaxation time of a fixed volume magnetic nano-particle is strongly dependent on the temperature as well as the effective magnetic anisotropy constant. Thermal stability of the magnetic particle implies large values of the relaxation time. A typical value accepted by the industry corresponds to a magnetic data storage medium thermally stable for $10 \mathrm{yr}$, so $\tau \approx 10 \mathrm{yr}^{1,2}$ This relaxation time is obtained when the ratio $\mathrm{K}_{\mathrm{a}}{ }^{\text {eff }} \mathrm{V} / \mathrm{k}_{\mathrm{b}} \mathrm{T}$ in the exponent of Eq. (5) is at least 40 or higher. ${ }^{1}$

We simulated RT thermal stability of multiferroic composite magnetic data storage media consisting of ferromagnetic nano-particles of sizes within the super-paramagnetic range, deposited onto a piezoelectric substrate. The thermal stability of super-paramagnetic nano-particles is established by activating the elasto-mechanical coupling between the nano-particles and the piezoelectric substrate through an applied voltage. As discussed earlier, for a negative applied voltage this has the effect of increasing the effective magnetocrystalline anisotropy constant when the magneto-striction
TABLE II. Simulation parameters used for two different magnetic materials with positive magneto-striction coefficients $\left(\lambda_{\mathrm{s}}>0\right)$.

\begin{tabular}{lcccc}
\hline \hline Material & $\lambda_{\mathrm{s}}$ & $\mathrm{K}_{\mathrm{a}}\left(\mathrm{J} / \mathrm{m}^{3}\right)$ & $\mathrm{Y}(\mathrm{GPa})$ & References \\
\hline $\mathrm{FePt}$ & $10 \times 10^{-5}$ & $6 \times 10^{7}$ & 180 & 23 \\
$\mathrm{Co}_{50} \mathrm{Fe}_{50}$ & $6.5 \times 10^{-5}$ & $1.5 \times 10^{4}$ & 200 & 24 \\
\hline \hline
\end{tabular}

and piezoelectric coefficients have opposite signs (see Table I). Assuming negative applied voltages throughout and the piezoelectric substrate $\mathrm{PbZrTiO}_{3}$ (PZT), which has typically $\mathrm{d}_{31}<0$, then the remaining choice of magnetic materials are those with positive saturation magneto-striction coefficient, $\lambda_{\mathrm{s}}>0$. Although there are many materials meeting this requirement, we have identified two suitable magnetic materials for the purpose of computational testing of this concept. These are listed in Table II together with their main parameters used in the calculations.

These materials have been have been selected because both $\mathrm{Fe}$ and Co alloys are usually utilized in magnetic data storage thin films. In addition to the parameters listed in Table II, we assumed in the simulations a PZT piezoferroelectric layer of $t_{e}=10 \mathrm{~nm}$ thickness with piezoelectric parameter of $\mathrm{d}_{31}=-1.7 \times 10^{-10} \mathrm{~m} / \mathrm{V}$.

Figures 2 and 3 show the thermal stability ratio $\mathrm{K}_{\mathrm{a}}{ }^{\text {eff }} \mathrm{V} /$ $\mathrm{k}_{\mathrm{b}} \mathrm{T}$ at room temperature $(\mathrm{T} \approx 300 \mathrm{~K})$ as a function of the magnetic grain radius for FePt and $\mathrm{CoFe}$ grains on PZT substrate, respectively.

Thermal stability ratio is plotted with and without electrically induced elasto-mechanical coupling effect (negative voltage). In the case of FePt, it can be seen that a magnetic recording medium stable for $10 \mathrm{yr}\left(\mathrm{K}_{\mathrm{a}}{ }^{\text {eff }} \mathrm{V} / \mathrm{k}_{\mathrm{b}} \mathrm{T} \approx 40\right)$ is obtained for a grain radius of about $1.9 \mathrm{~nm}$.

When the system is electrically activated by $-10 \mathrm{~V}$ applied voltage, thermally stable medium is achieved for grains as small as $1.5 \mathrm{~nm}$ radius. Calculations indicate that the effective magneto-crystalline anisotropy is $70 \%$ larger for FePt grains on PZT when $-10 \mathrm{~V}$ electric field is applied (see relation (4)).

\section{FePt nano grains / PZT}

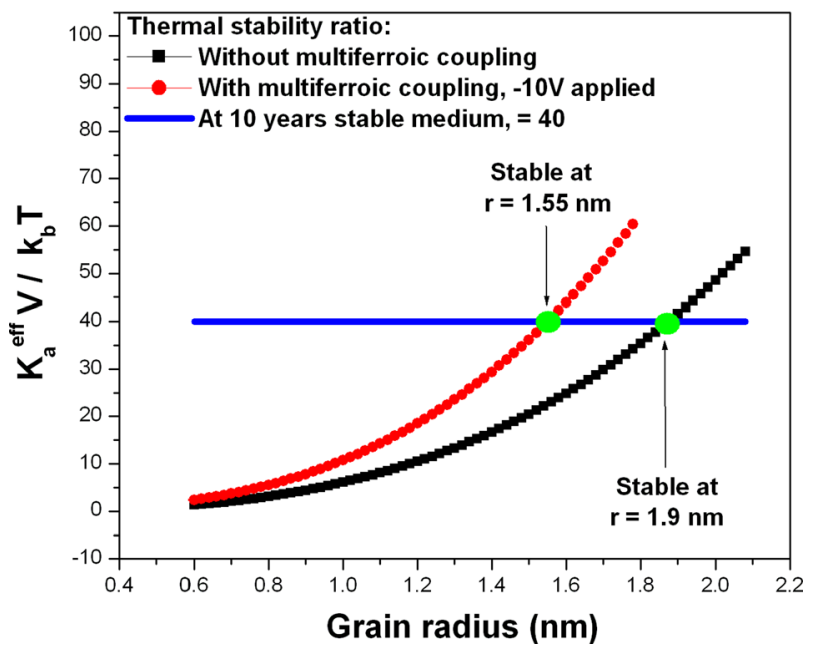

FIG. 2. Thermal stability ratio of a magnetic recording medium consisting of FePt grains on PZT, with and without electrically induced magnetoelectric coupling. 
This increase in the effective magneto-crystalline anisotropy is indeed responsible for the improvement of thermal stability, which leads to further increases in the areal magnetic storage density.

Figure 3 shows the same data for CoFe nano-grains. In this case, the effect of the electrically induced elastomechanical coupling is more substantial than in the case of FePt because of the lower magneto-crystalline anisotropy of $\mathrm{CoFe}$. Without electrical excitation, thermal stability criteria require much larger $\mathrm{CoFe}$ grains of about $14 \mathrm{~nm}$ radius. Quite remarkably, the application of $-1 \mathrm{~V}$ electric field equivalent is sufficient to achieve thermally stable grains as small as $4.8 \mathrm{~nm}$ radius. The application of $-1 \mathrm{~V}$ results in an effective magneto-crystalline anisotropy 23 times larger than that of the CoFe itself $\left(\mathrm{K}_{\mathrm{a}}^{\mathrm{eff}} \approx 23 \times \mathrm{K}_{\mathrm{a}}\right)$.

These results clearly indicate the huge potential of this technology to push the boundaries of magnetic data storage beyond the super-paramagnetic limit.

\section{MAGNETIC RECORDING PROCEDURE}

Although we discussed this concept in the context of increasing the effective magneto-crystalline anisotropy via an applied voltage, the same effect can work in reverse (see Table I). Hence, the effective magneto-crystalline anisotropy can also be lowered by the application of a voltage (see Table I). Interestingly, both possible cases of this effect are applicable to magnetic data storage. The lowering of magneto-crystalline anisotropy when a voltage is applied could be used to replace HAMR technology, while the increase of the effective magneto-crystalline anisotropy could be used to further decrease the grain size of low anisotropy magnetic materials without losing thermal stability.

In order to implement technologically this concept, two possible recording protocols are proposed.

Figure 4 shows the magnetic recording "write" protocol proposed for the replacement of HAMR technology when ultra-high magneto-crystalline anisotropy materials are utilized to store data.

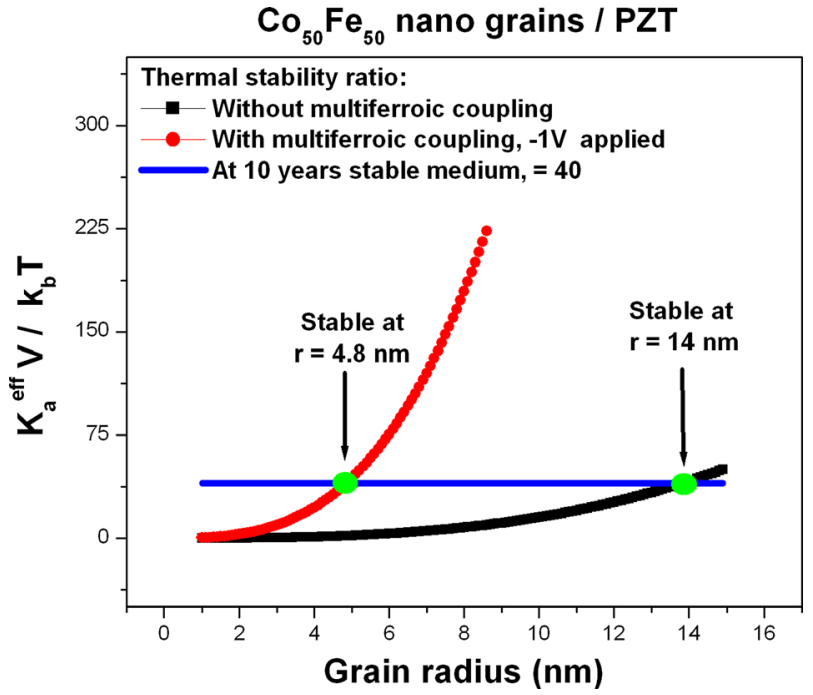

FIG. 3. Thermal stability ratio of a magnetic recording medium consisting of $\mathrm{CoFe}$ grains on PZT, with and without electrically induced magnetoelectric coupling.

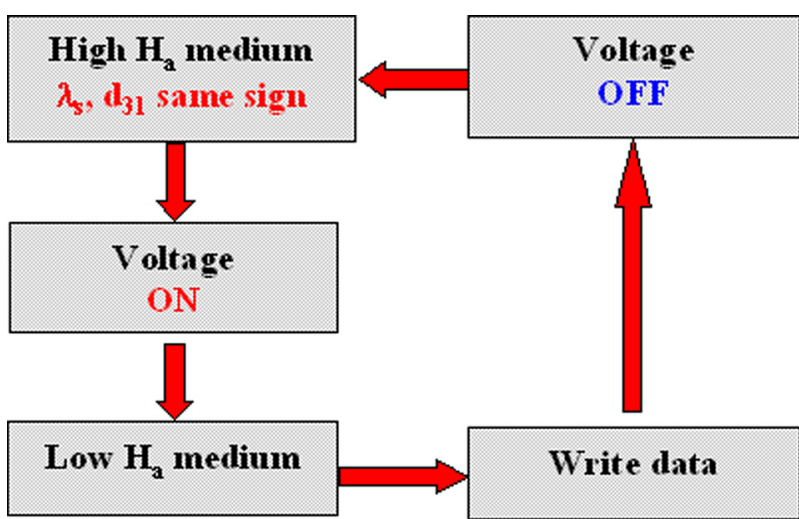

FIG. 4. Magnetic data storage "write" protocol for multiferroic coupled magnetic recording medium with ultra high magnetic anisotropy. Scheme to replace HAMR.

This case requires the application of a suitable negative voltage only during the "write" process, after which the removal of the electric field will result in grains returning to high anisotropy, highly stable state.

Figure 5 shows the opposite case, in which small magneto-crystalline anisotropy materials are utilized. In this case, very small grains become thermally unstable and the present multiferroic-coupling scheme is used to artificially increase the magneto-crystalline effective anisotropy.

The write protocol involves, in this case, the lowering or removal of the applied negative voltage followed by the "write" process and the return to the suitable applied negative voltage.

Unlike the previous scheme that requires the applied voltage only during the write process, this second protocol requires a voltage permanently applied.

The implementation of these protocols is very challenging and requires additional scientific and engineering work.

Although we take the view that the both protocols could be implemented by addressing electrically the whole wafer/ disk rather than individual magnetic bits, this is particularly more feasible for the protocol discussed in diagram 5, but

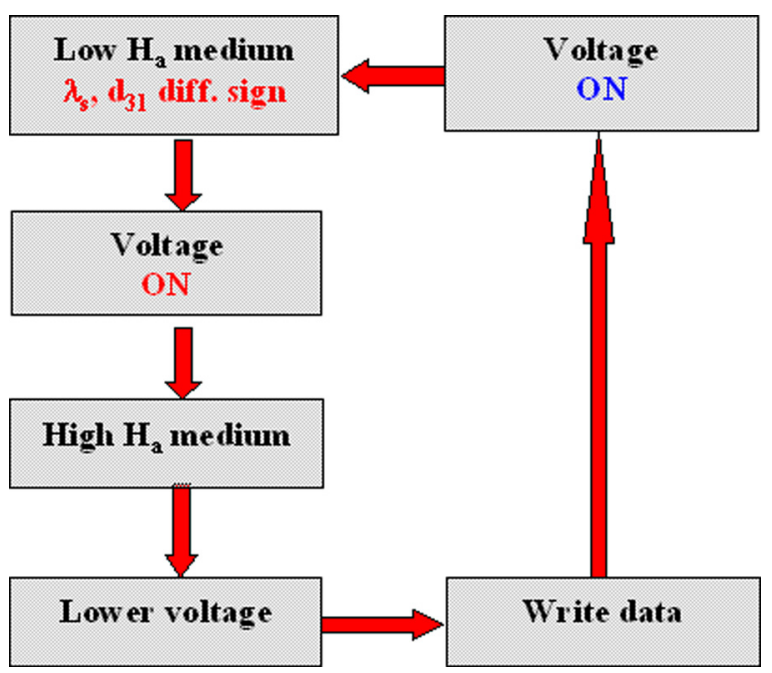

FIG. 5. Thermal stability ratio of a magnetic recording medium consisting of CoFe grains on PZT, with and without electrically induced magnetoelectric coupling. 
less obvious for the scheme proposed to replace HAMR. We therefore hope that this work will stimulate experimental investigation of these concepts with possible far reaching consequences for magnetic data storage industry.

\section{CONCLUSION}

There is clear theoretical evidence that electrically induced elasto-mechanical coupling could be effectively applied to develop multiferroic composite magnetic recording media. We demonstrated that the elasto-mechanical coupling significantly alters the effective magneto-crystalline anisotropy of magnetic grains when they are coupled to a piezo-electric substrate. A formula describing the effective magneto-crystalline anisotropy of a multiferroic coupled magnetic particle has been derived. The theory predicts that, for negative applied voltages, when $\lambda_{\mathrm{s}}$ and $d_{31}$ have the same sign, a decrease in the magneto-crystalline anisotropy is observed. When $\lambda_{\mathrm{s}}$ and $\mathrm{d}_{31}$ have opposite signs, an increase in the effective magneto-crystalline anisotropy is achieved. At positive applied voltages, the effect is fully reversed giving additional versatility in choosing suitable materials. These two cases could be potentially utilized either as an alternative to HAMR technology, or to further increase the magnetic storage density beyond the super-paramagnetic limit.

${ }^{1}$ D. Weller and A. Moser, IEEE Trans. Magn. 35(6), 4423-4439 (1999).

${ }^{2}$ K. Matsumoto, A. Inomata, and S.-Y. Hasegawa, Fujitsu Sci. Tech. J. 42, 158-167 (2006).

${ }^{3}$ R. E. Rottmayer, S. Batra, D. Buechel, W. A. Challener, J. Hohlfeld, Y. Kubota, L. Li, B. Lu, C. Mihalcea, K. Mountfield, K. Pelhos, C. Peng, T. Rausch, M. A. Seigler, D. Weller, and X. M. Yang, IEEE Trans. Magn. 42(10), 2417-2421 (2006).
${ }^{4}$ H. Katayama, S. Sawamura, Y. Ogimoto, J. Nakajima, K. Kojima, and K. Ohta, J. Magn. Soc. Jpn. 23(S1), S1_233 (1999).

${ }^{5}$ H. Saga, H. Nemoto, H. Sukeda, and M. Takahashi, Jpn. J. Appl. Phys., Part 1 38, 1839 (1999).

${ }^{6}$ K.-S. Park, Y.-P. Park, and N.-C. Park, IEEE Trans. Magn. 47(3), 539-545 (2011).

${ }^{7}$ V. Skumryev, S. Stoyanov, Y. Zhang, G. Hadjipanayis, D. Givord, and J. Nogues, Nature 423, 850-853 (2003).

${ }^{8}$ J. Eisenmenger and I. K. Schuller, Nat. Mater. 2, 437-438 (2003).

${ }^{9}$ E. E. Fullerton, D. T. Margulies, M. E. Schabes, M. Carey, B. Gurney, A. Moser, M. Best, G. Zeltzer, K. Rubin, H. Rosen, and M. Doerner, Appl. Phys. Lett. 77, 3806 (2000).

${ }^{10}$ H. K. D. Kim, L. T. Schelhas, S. Keller, J. L. Hockel, S. H. Tolbert, and G. P. Carman, Nano Lett. 13, 884-888 (2013).

${ }^{11}$ R. Sbiaa and S. N. Piramanayagam, Recent Pat. Nanotechnol. 1, 29-40 (2007).

${ }^{12}$ C. W. Nan, M. I. Bichurin, S. Dong, D. Viehland, and G. Srinivasan, J. Appl. Phys. 103(3), 031101 (2008).

${ }^{13}$ G. Srinivasan, Annu. Rev. Mater. Res. 40, 153-178 (2010).

${ }^{14}$ Y. Wang, J. Hu, Y. Lu, and C.-W. Nan, NPG Asia Mater. 2(2) 61-68 (2010).

${ }^{15}$ J. Zhai, Z. Xing, S. Dong, J. Li, and D. Viehland, J. Am. Ceram. Soc. 91(2) 351-358 (2008).

${ }^{16}$ M. Vopsaroiu, M. Stewart, T. Hegarty, A. Muniz-Piniella, N. McCartney, M. Cain, and G. Srinivasan, Meas. Sci. Technol. 19, 045106 (2008).

${ }^{17}$ T. Jin, L. Hao, J. Cao, M. Liu, H. Dang, Y. Wang, D. Wu, J. Bai, and F. Wei, Appl. Phys. Express 7, 043002 (2014).

${ }^{18}$ S. Saranu, S. Selve, U. Kaiser, L. Han, U. Wiedwald, P. Ziemann, and U. Herr, Beilstein J. Nanotechnol. 2, 268-275 (2011).

${ }^{19}$ R. Streubel, D. Köhler, R. Schäfer, and L. M. Eng, Phys. Rev. B 87, 054410 (2013).

${ }^{20}$ A. Sukhov, L. Chotorlishvili, P. P. Horley, C.-L. Jia, S. K. Mishra, and J. Berakdar, J. Phys. D: Appl. Phys. 47, 155302 (2014).

${ }^{21}$ S. Giordano, Y. Dusch, N. Tiercelin, P. Pernod, and V. Preobrazhensky, J. Phys. D: Appl. Phys. 46, 325002 (2013).

${ }^{22}$ C. Jia, A. Sukhov, P. P. Horley, and J. Berakdar, EPL 99, 17004 (2012).

${ }^{23}$ P. Rasmussen, X. Rui, and J. E. Shield, Appl. Phys. Lett. 86, 191915 (2005).

${ }^{24}$ M. D. Cooke, L.-C. Wang, R. Watts, R. Zuberek, G. Heydon, W. M. Rainforth, and G. A. Gehring, J. Phys. D: Appl. Phys. 33, 1450-1459 (2000). 